\title{
El Comportamiento Electoral Municipal en El Salvador
}

\section{Efectos de la simultaneidad de las elecciones legislativas y municipales.}

En El Salvador la elección de los Concejos Municipales coincide en hora y fecha con la elección de diputados a la Asamblea Legislativa. Ambas elecciones se llevan a cabo cada tres años. Este hecho es el que posibilita hablar de simultaneidad electoral. Sin embargo, decir que el electorado puede votar simultáneamente para dos elecciones diferentes no quiere decir que necesariamente su voto es coincidente ni, mucho menos, que el resultado electoral sigue la misma suerte. Hay una serie de elementos de los sistemas electorales para cada ámbito (municipal y legislativo) que posibilitan resultados diversos. Por ejemplo, el uso de una papeleta de votación para cada tipo de elección, en la cual los partidos contendientes no tienen por qué ser los mismos. La utilización de diferentes papeletas posibilita, a su vez, dos lógicas de votación distintas. Los electores pueden votar, si así lo desean, por partidos diferentes en cada elección aun cuando los partidos contendientes fuesen los mismos. Es decir, es posible que los electores emitan un voto cruzado.

¿Qué efectos tiene esta simultaneidad de procesos electorales? ¿En qué medida la simultaneidad de procesos electorales de diverso ámbito afecta los resultados que se obtienen en cada uno de ellos? ¿La lógica de la disputa por el poder local (municipal) está condicionada por la lógica de la disputa por el poder al nivel nacional (legislativo)? Para intentar responder estas preguntas utilizaré los resultados electorales de 1994, 1997 y 2000 tanto al nivel municipal como a nivel departamental. Dado que tres procesos electorales no son suficientes para establecer claras tendencias (estables en el tiempo), los hallazgos que presento deben ser considerados como provisionales, como hipótesis fundamentadas empíricamente para posteriores investigaciones'. 


\section{1 ¿Dos elecciones bajo una misma lógica?}

Los ciudadanos que participan en ambos niveles de elecciones podrían hacerlo bajo dos lógicas distintas (voto cruzado) ${ }^{2}$ o emitiendo sus votos para el mismo partido (sí éste compite en ambos niveles). En el primero de los casos planteados no habría duda sobre la existencia de una lógica municipal (local) y otra, de carácter nacional ${ }^{3}$. Denotaría incluso un comportamiento electoral racional. En el segundo caso probablemente estaríamos frente a un comportamiento electoral, bien por identificación partidista, bien por simpatía con la candidatura, o bien por tradición o costumbre.

La existencia, o no, de una o dos lógicas de votación podría expresarse también en los niveles de concurrencia para ambas elecciones. Una concurrencia diferenciada podría estar manifestando la presencia de dos lógicas de votación. Es decir, dado que se celebran elecciones locales y nacionales de forma simultánea, una diferencia significativa en los niveles de votación entre ambos niveles estaría expresando, de forma agregada, dos comportamientos electorales distintos. Los electores se habrían decidido a votar en un nivel electoral pero abstenerse en el otro. Una situación así sería perfectamente posible en El Salvador dado el uso de papeletas de votación diferentes según se trate de la elección local o la elección nacional.

¿A quién puede importarle saber si existe una o dos lógicas de votación en nuestro país? Inicialmente puede ser de interés para los partidos políticos que compiten por los votos de los electores. La existencia de una lógica puede ahorrarles costos en su campaña electoral porque concentrarían sus esfuerzos en la dirección de la lógica existente. De hecho, los partidos políticos juegan al "efecto arrastre" del voto desde un nivel hacia el otro; o desde un candidato o candidata hacia el partido". Otros interesados pueden ser los llamados "analistas" políticos. Así tendrían más argumentos y herramientas para afinar o hacer más riguroso el análisis. También puede interesar a la máxima autoridad en materia electoral, bien para organizar adecuadamente el proceso, o bien, porque tal vez podría ser un elemento a considerar al momento de presupuestar el evento. En este último caso podríamos preguntarnos por la conveniencia, o no, de emitir dos papeletas de votación cuando el efecto agregado es como si sólo se necesitara una papeleta válida para los dos niveles de elección ${ }^{5}$. Finalmente podría también ser de interés para la ciudadanía interesada en democratizar aún más nuestro régimen político. Si se puede demostrar la existencia de dos lógicas de votación podría promoverse la necesidad de abrir nuestro sistema electoral de tal forma que se facilite la existencia de partidos de ámbito local ${ }^{h}$.

Comenzaré a dilucidar la existencia de una o dos lógicas de votación en El Salvador analizando las diferencias en votos válidos para los dos niveles de elección. El Cuadro № 1 muestra la información pertinente considerando los votos en cada departamento para los tres años electorales aquí considerados ${ }^{7}$. Si 
consideramos que los votos válidos registrados para el nivel local representan los votos válidos esperados para el nivel nacional, bajo la hipótesis de una misma lógica de votación, podemos llevar a cabo una prueba de $c^{2}$ y establecer si las diferencias que se observan entre pares de columnas son o no significativas.

\section{Cuadro № 1}

Comparación del voto "local” y "nacional", según Departamentos $-1994,1997$ y 2000.

\begin{tabular}{l|rr|rr|rr}
\hline \multirow{2}{*}{ Departamento } & \multicolumn{7}{|c|}{ VOTOS VÁLIDOS } \\
& \multicolumn{2}{|c|}{1994} & \multicolumn{2}{c}{1997} & \multicolumn{2}{c}{2000} \\
\cline { 2 - 7 } & \multicolumn{1}{|c|}{ Local } & Nacional & \multicolumn{1}{|c}{ Local } & Nacional & Local & Nacional \\
\hline San Salvador & 417,404 & 421,206 & 340,617 & 341,286 & 370,910 & 363,846 \\
Santa Ana & 133,710 & 133,667 & 106,327 & 106,941 & 101,152 & 101,198 \\
San Miguel & 84,800 & 83,605 & 76,863 & 76,741 & 85,017 & 84,830 \\
La Libertad & 152,458 & 151,816 & 120,759 & 120,771 & 132,256 & 132,191 \\
Usulután & 72,266 & 71,787 & 60,975 & 60,874 & 71,529 & 71,297 \\
Sonsonate & 106,107 & 106,043 & 82,891 & 82,927 & 91,407 & 91,377 \\
La Unión & 46,115 & 45,969 & 39,835 & 39,753 & 43,593 & 43,402 \\
La Paz & 68,635 & 68,329 & 59,347 & 59,341 & 66,003 & 66,196 \\
Chalatenango & 50,613 & 50,252 & 44,839 & 44,879 & 51,037 & 50,882 \\
Cuscatlán & 48,695 & 48,602 & 41,972 & 41,731 & 44,903 & 44,960 \\
Ahuachapán & 60,497 & 60,388 & 53,331 & 53,087 & 58,091 & 58,223 \\
Morazán & 36,126 & 35,812 & 33,402 & 33,410 & 39,915 & 39,817 \\
San Vicente & 37,545 & 37,426 & 33,068 & 32,805 & 32,868 & 32,804 \\
Cabañas & 30,483 & 30,375 & 25,063 & 25,057 & 29,315 & 29,246 \\
\hline Totales & $1,345,454$ & $1,345,277$ & $1,119,289$ & $1,119,603$ & $1,217,996$ & $1,210,269$ \\
\hline
\end{tabular}

Fuentes: elaboración propia sobre resultados oficiales.

Para 1994 y 1997, en TSE (2000)

Para 2000, en ECA \# 617.

Procediendo de la manera indicada, con un nivel de significancia del $5 \%$, los resultados del análisis son:

a) Las diferencias son significativas en 1994 y 2000 pero no en 1997. Esto nos estaría indicando que el voto no es independiente del tipo de elección. Al menos al nivel agregado pareciéramos estar ante dos lógicas de votación ${ }^{k}$.

b) Si se excluyen los departamentos de San Salvador y San Miguel para el año 1994, las diferencias dejan de ser entonces significativas. Esto nos estaría indicando que en 12 de los 14 departamentos el electorado actúa bajo una sola lógica de votación. 
c) Si se excluye el departamento de San Salvador para el año 2000, las diferencias tampoco son significativas. Esto quiere decir que en 13 de los 14 departamentos del país, el electorado sigue una sola lógica de votación.

d) Las tres observaciones anteriores sugieren una tendencia a la disminución de las diferencias en votos válidos según el tipo de elección. Al seguir las observaciones b) y c) resulta que, estadísticamente hablando, el c c calculado se hace cada vez más pequeño.

Otra forma de aproximamos a la identificación de la existencia de una o dos lógicas de participación electoral consiste en un análisis de correlación entre votos locales y nacionales. Si hubiese una correlación alta, significaría que los electores siguen una misma lógica. Una elección "arrastra" a la otra. El electorado no discrimina entre elecciones. Pues bien, el análisis de correlación sugiere exactamente este comportamiento dado que el coeficiente de correlación de Pearson asume un valor muy cercano o igual a 1 (0.999 para 1994; 1.000 para 1997 y 0.999 para 2000).

Antes de seguir examinando la existencia de una o dos lógicas de votación, la información del Cuadro № 1 también nos permite captar otras características que describen el comportamiento del electorado salvadoreño en elecciones locales. En primer lugar, las elecciones de 1994 fueron las de mayor concurrencia efecti$v a^{9}$. Este fenómeno estaba en consonancia con las expectativas derivadas del hecho de ser las primeras elecciones realmente libres y competitivas en más de 60 años y las primeras elecciones después de instaurado un nuevo régimen político en El Salvador '" . Además, si consideramos que justamente en 1994 la simultaneidad abarcó a las elecciones presidenciales podría pensarse que la mayor concurrencia en este año esté asociada al "arrastre" que la elección presidencial implicaba.

En segundo lugar, llama poderosamente la atención el descenso participativo entre los seis años que van de 1994 a 2000 . Cerca de 127 mil votos efectivos menos. Si suponemos que en dicho período el potencial electorado se habría incrementado por razones demográficas, lo menos que se podía esperar era un incremento de votación correspondiente al crecimiento "vegetativo" del electorado. Sin embargo, no sólo no hubo incremento sino que la votación efectiva descendió.

El Cuadro № 1 utiliza como base de datos los totales de votos válidos para cada departamento. Esto quiere decir que si las diferencias entre votos locales y votos nacionales no son significativas, los votos municipales se agregan de tal forma que "igualan" a los votos nacionales. Pareciera entonces que la lógica predominante sería de carácter nacional. Las elecciones legislativas terminarían arrastrando a las elecciones municipales. La lógica de la competencia sería nacional antes que municipal. Pero, ¿qué pasa si utilizamos los votos municipales para llevar a cabo el análisis? El Cuadro $\mathrm{N}^{0} 2$ presenta la información para seguir esta otra vía de exploración del problema de una o dos lógicas en el comportamiento electoral. Se trata de una exploración por cuanto apenas estoy considerando 14 de los 262 municipios. Siguiendo un criterio administrativo he 
optado por analizar lo que ocurre en las 14 cabeceras departamentales. Los votos locales son los votos válidos para elecciones de concejos municipales registrados en la cabecera departamental en cada año electoral. Los votos nacionales son los votos válidos para elecciones de diputados registrados también en las cabeceras"

A simple vista las diferencias de votos para cada par de columnas parecen pequeñas. El análisis de $\chi^{2}$ para estas diferencias determina que no son significativas $(a=0.05)$. No ha sido necesario excluir a ningún municipio para llegar a esta conclusión como lo era en el caso del análisis al nivel de departamentos. Parece claro, entonces, que el electorado sigue una misma lógica para la concurrencia efectiva. Ahora bien, ¿es una lógica nacional o se trata de una lógica local? ¿Son las elecciones nacionales las que arrastran a las elecciones locales o es a la inversa? Al haber realizado el análisis tomando en cuenta los votos en los municipios podría plantearse que es la elección local la que arrastra a la nacional. Justamente una conclusión contraria a la planteada a partir del análisis del Cuadro № 1 . Por tanto, si bien podemos sostener empíricamente la existencia de una sola lógica de votación, no podemos establecer con esta información a qué ámbito obedece esta lógica.

Cuadro No. 2

Comparación del voto "local" y "nacional”, según Municipios $-1994,1997$ y 2000 -

\begin{tabular}{|c|c|c|c|c|c|c|}
\hline \multirow{3}{*}{ Municipios } & \multicolumn{6}{|c|}{ VOTOS VÁLIDOS } \\
\hline & \multicolumn{2}{|c|}{1994} & \multicolumn{2}{|c|}{1997} & \multicolumn{2}{|c|}{2000} \\
\hline & Local & Nacional & Local & Nacional & Local & Nacional \\
\hline San Salvador & 60,793 & 161,381 & 120,839 & 122,002 & 121,923 & 122,738 \\
\hline Santa Ana & 67,085 & 67,202 & 51,668 & 51,703 & 45,495 & 45,512 \\
\hline San Miguel & 40,989 & 40,950 & 35,597 & 35,515 & 37,230 & 37,182 \\
\hline Nueva San Salvador & 40,262 & 39,935 & 31,306 & 31,356 & 35,059 & 35,034 \\
\hline Usulután & 18,374 & 18,236 & 13,986 & 13,933 & 14,595 & 14,543 \\
\hline Sonsonate & 27,702 & 27,643 & 19,676 & 19,702 & 20,413 & 20,452 \\
\hline La Unión & 11,402 & 11,349 & 9,253 & 9,269 & 8,648 & 8,571 \\
\hline Zacatecoluca & 18,350 & 18,221 & 15,197 & 15,323 & 15,756 & 15,806 \\
\hline Chalatenango & 7,691 & 7,686 & 7,074 & 7,040 & 8,129 & 8,161 \\
\hline Cojutepeque & 16,530 & 16,534 & 13,582 & 13,542 & 11,794 & 11,802 \\
\hline Ahuachapán & 21,019 & 20,980 & 18,164 & 18,059 & 18,286 & 18,406 \\
\hline San Francisco Gotera & 4,951 & 4,921 & 4,411 & 4,409 & 4,985 & 4,927 \\
\hline San Vicente & 14,976 & 14,971 & 12,583 & 12,531 & 10,527 & 10,487 \\
\hline Sensuntepeque & 9,174 & 9,085 & 6,801 & 6,801 & 7,115 & 7,107 \\
\hline
\end{tabular}

Fuentes: claboración propia sobre resultados oficiales.

Para 1994 y 1997, en TSE (2000)

Para 2000, en ECA \# 617 


\section{2 ¿Votando al mismo partido independientemente del tipo de elección?}

La existencia de una sola lógica para acudir o no a las umas de votación no nos permite saber si, en términos agregados, los electores votan o no al mismo partido. Para intentar arrojar luz sobre este "misterio" analizaré si las diferencias entre los votos que reciben los partidos en cada tipo de elección son o no significativas. Si las diferencias no son significativas (con un $\alpha=0.05$ ) resultará verosímil el que los electores votan al mismo partido independientemente del tipo de elección. Utilizaré para este análisis los votos por ARENA, FMLN y PCN en las elecciones de concejos municipales de 2000. De nuevo restringiré el análisis a lo ocurrido en las catorce cabeceras del país. La información la presento en el Cuadro № 3.

Al hacer el análisis de $\chi^{2}$, bajo la hipótesis de independencia del tipo de elección, las diferencias resultan significativas. Esto quiere decir que, al menos para los partidos y municipios analizados, los electores siguen lógicas distintas al momento de dar su voto por un partido. En otras palabras, estamos en presencia de un voto cruzado. Así que, pese a existir una sola lógica para concurrir a votar (quien vota para un nivel, vota para el otro), al momento de decidir por quién votar, muchos quienes votan por un partido en un nivel votan por otro partido en el otro nivel. El efecto agregado implica dos lógicas de votación.

\section{Cuadro № 3}

Comparación del voto "local" y "nacional"

por partido, según municipios

-elecciones 2000-

\begin{tabular}{|c|c|c|c|c|c|c|}
\hline \multirow{3}{*}{ Municipios } & \multicolumn{6}{|c|}{ VOTOS VÁLIDOS } \\
\hline & \multicolumn{2}{|c|}{1994} & \multicolumn{2}{|c|}{1997} & \multicolumn{2}{|c|}{2000} \\
\hline & Local & Nacional & Local & Nacional & Local & Nacional \\
\hline San Salvador & 47,575 & 49,809 & $* 68,660$ & 52,243 & 1,619 & 2,102 \\
\hline Santa Ana & 15,131 & 16,183 & 14,702 & 17,461 & 2,023 & 2,002 \\
\hline San Miguel & 9,207 & 9,912 & 7,435 & 10,746 & 1,082 & 1,473 \\
\hline Nueva San Salvador & 13,194 & 13,071 & 16,112 & 15,219 & 1,368 & 1,044 \\
\hline Usulután & 5,019 & 4,989 & $* 2,878$ & 4,165 & 5,419 & 3,621 \\
\hline Sonsonate & 7,655 & 7,411 & 8,540 & 8,023 & 713 & 753 \\
\hline La Unión & 4,378 & 3,630 & 486 & 1,184 & 443 & 954 \\
\hline Zacatecoluca & 4,675 & 4,594 & 7,531 & 7,310 & 1,735 & 1,554 \\
\hline Chalatenango & 3,407 & 3,370 & 3,957 & 3,666 & 178 & 197 \\
\hline Cojutepeque & 4,156 & 4,077 & 3,365 & 3,382 & 1,172 & 917 \\
\hline Ahuachapán & 6,283 & 5,930 & 6,564 & 6,068 & 3,017 & 3,075 \\
\hline San Francisco Gotera & 1,722 & 1,524 & $* 1,785$ & 1,101 & 924 & 1,078 \\
\hline San Vicente & 4,399 & 4,415 & 4,062 & 3,813 & 733 & 756 \\
\hline Sensuntepeque & 2,343 & 2,479 & 1,692 & 1,578 & 1,215 & 1,104 \\
\hline
\end{tabular}

* Coalición encabezada por el FMLN.

Fucntc: claboración propia sobre resultados oficiales en ECA \# 617. 
Al observar con mayor detenimiento el Cuadro $\mathrm{N}^{2} 3$ podemos darnos cuenta que la existencia de dos lógicas de votación, al considerar todos los municipios, podría estarse reflejando en el hecho de que no siempre los partidos reciben más votos en el mismo tipo de elección. Es decir, en algunos municipios los partidos reciben más votos en elecciones locales mientras que en otros, los reciben más en elecciones nacionales ${ }^{12}$. Para llevar a cabo este análisis sobre la información del Cuadro № 3 podríamos tener en cuenta que:

a) Si los votos locales son más que los votos nacionales, estamos ante el predominio de una lógica local. La disputa por el poder local cobra mayor importancia para los electores quienes al nivel nacional pueden votar por otro partido ${ }^{13}$.

b) Si los votos nacionales son más que los votos locales, estamos ante el predominio de una lógica nacional. La disputa por los escaños legislativos es más relevante para los electores quienes al nivel municipal pueden votar por otro partido ${ }^{14}$.

Según los criterios arriba establecidos tenemos que para los electores que en marzo del 2000 dieron su voto por ARENA en San Salvador, Santa Ana, San Miguel, San Vicente y Sensuntepeque la elección nacional era más importante; lo mismo diríamos para quienes dieron su voto por el FMLN en Santa Ana, San Miguel, Usulután, La Unión y Cojutepeque; de igual manera para quienes dieron su voto por el PCN en San Salvador, San Miguel, Sonsonate, La Unión, Chalatenango, Ahuachapán, San Francisco Gotera y San Vicente.

El voto local habría predominado para quienes votaron por ARENA, en Nueva San Salvador, Usulután, Sonsonate, La Unión, Zacatecoluca, Chalatenango, Cojutepeque, Ahuachapán y San Francisco Gotera; por el FMLN, en San Salvador, Nueva San Salvador, Sonsonate, Zacatecoluca, Chalatenango, Ahuachapán, San Francisco Gotera, San Vicente y Sensuntepeque; por el PCN, en Santa Ana, Nueva San Salvador, Usulután, Zacatecoluca, Cojutepeque y Sensuntepeque.

Considerando únicamente a estos partidos podría concluirse que la disputa en Nueva San Salvador y Zacalecoluca fue predominantemente local. En estos municipios, los tres partidos obtuvieron más votos locales que nacionales. La disputa se centraba por el concejo municipal. Por otro lado, la disputa habría tenido tintes predominantemente nacionales en San Miguel. Los tres partidos obtuvieron su mayor cantidad de votos en el ámbito nacional. En el resto de municipios la situación es heterogénea.

Otra forma de estudiar el predominio de una u otra lógica de votación en el electorado de cada uno de los partidos es justamente atendiendo la heterogeneidad $u$ homogeneidad del voto por partido en cada municipio. Para ello podemos valernos del índice de heterogeneidad del voto (HeV) cuyo valor nos indica cuán heterogéneo es el comportamiento electoral de los votantes. Cuanto mayor sea el valor de dicho índice, mayor heterogeneidad habrá. Esto a su vez indicaría una situación donde tiende a prevalecer una doble lógica de votación que, en 
definitiva, nos mostraría cuán fluido es el voto por los partidos en el municipio analizado $^{15}$. Los valores de $\mathbf{H e V}$ se muestran en el Cuadro $\mathrm{N}^{2} 4$ para cada municipio. También se muestra el valor promedio para los catorce municipios.

Como puede notarse el voto por ARENA resulta ser más homogéneo comparado con el voto por el FMLN y PCN. La heterogeneidad del primero es más baja que la de los otros dos partidos. Dicho de otra forma, quienes votan por ARENA en un nivel tienden, en general y de manera agregada, a votarle en el otro nivel. No sucede así en el caso del FMLN y el PCN. Si tomamos en cuenta que la heterogeneidad del voto nos indica también la fluidez del voto, los valores del Cuadro №4 indican que ARENA tiene un voto más consolidado. Lo cual quiere decir que su voto proviene normalmente de sus seguidores. No se nutre tanto de votantes de otros partidos o de indecisos. Sus electores le prefieren en ambos niveles. El FMLN y el PCN tienden a nutrirse de votantes ajenos o de indecisos según lo que esté en juego y según sean las percepciones de éstos en cada nivel electoral.

\section{Cuadro № 4}

Heterogeneidad del voto, por partido y municipio elecciones 2000 -porcentajes-

\begin{tabular}{l|r|r|r}
\hline \multirow{2}{*}{\multicolumn{1}{c|}{ Municipios }} & \multicolumn{2}{|c}{ Índice de Heterogeneidad del Voto } \\
\cline { 2 - 4 } & ARENA & FMLN & PCN \\
\hline San Salvador & 4.6 & 27.2 & 26.0 \\
Santa Ana & 6.7 & 17.2 & 1.0 \\
San Miguel & 7.4 & 36.4 & 30.6 \\
Nueva San Salvador & 0.9 & 5.7 & 26.9 \\
Usulután & 0.6 & 36.5 & 39.8 \\
Sonsonate & 3.2 & 6.2 & 5.5 \\
La Unión & 18.7 & 83.6 & 73.2 \\
Zacatecoluca & 1.7 & 3.0 & 11.0 \\
Chalatenango & 1.1 & 7.6 & 10.1 \\
Cojutepeque & 1.9 & 0.5 & 24.4 \\
Ahuachapán & 5.8 & 7.9 & 1.9 \\
San Francisco Gotera & 12.2 & 47.4 & 15.4 \\
San Vicente & 0.4 & 6.3 & 3.1 \\
Sensuntepeque & 5.6 & 7.0 & 9.6 \\
\hline Promedio & 5.1 & 20.9 & 19.9 \\
\hline
\end{tabular}

Fuente: claboración propia. 
Finalmente el Cuadro № 5 presenta una clasificación de las distintas cabeceras departamentales según el índice $\mathrm{HeV}$ para cada partido. Este cuadro nos permite ver también la menor heterogeneidad en el comportamiento electoral de los votantes del partido ARENA. En ocho de las catorce cabeceras el HeV es muy bajo. Situación que contrasta con las cinco o seis cabeceras donde el $\mathrm{HeV}$ es muy alto para el FMLN y PCN respectivamente. Esto quiere decir que los votantes de ARENA, en esas ocho cabeceras tienden a votar bajo un mismo criterio en ambos tipos de elecciones. Su decisión resulta independiente si se trata de elecciones a concejos municipales o elecciones de diputados. Tienden a "marcar la crucita" en las dos papeletas de votación. El voto para el FMLN y PCN en San Salvador, San Miguel, Usulután y La Unión depende del tipo elección. Quienes votan por ellos no son absolutamente leales. Razonan su voto'th.

Cuadro № 5

Heterogeneidad de las cabeceras departamentales según voto por partido

\begin{tabular}{|c|c|c|c|}
\hline \multirow{2}{*}{$\begin{array}{c}\text { Índice HeV } \\
\text { (clasificación) }\end{array}$} & \multicolumn{3}{|c|}{ Cabeceras con similar heterogeneidad del voto } \\
\hline & ARENA & FMLN & PCN \\
\hline $\begin{array}{c}0-5 \\
\text { (muy baja) }\end{array}$ & $\begin{array}{c}\text { San Salvador } \\
\text { Nueva San Salvador } \\
\text { Usulután } \\
\text { Zacatecoluca } \\
\text { Chalatenango } \\
\text { Cojutepeque } \\
\text { San Vicente }\end{array}$ & $\begin{array}{l}\text { Zacatecoluca } \\
\text { Cojutepeque }\end{array}$ & $\begin{array}{c}\text { Santa Anta } \\
\text { San Francisco Gotera }\end{array}$ \\
\hline $\begin{array}{l}5.1-10 \\
\text { (baja) }\end{array}$ & $\begin{array}{c}\text { Santa Ana } \\
\text { San Miguel } \\
\text { Ahuachapán } \\
\text { Sensuntepeque }\end{array}$ & \begin{tabular}{|c|} 
Nueva San Salvador \\
Sonsonate \\
Chalatenango \\
Ahuachapán \\
San Vicente \\
Sensuntepeque
\end{tabular} & $\begin{array}{c}\text { Sonsonate } \\
\text { Sensuntepeque }\end{array}$ \\
\hline $\begin{array}{l}10.1-15 \\
\text { (mediana) }\end{array}$ & San Francisco Gotera & & $\begin{array}{l}\text { Zacatecoluca } \\
\text { Chalatenango }\end{array}$ \\
\hline $15.1-20$ (alta) & La Unión & Santa Ana & San Francisco Gotera \\
\hline $\begin{array}{l}\text { Más de } 20 \\
\text { (muy alta) }\end{array}$ & $\because$ & $\begin{array}{c}\text { San Salvador } \\
\text { San Miguel } \\
\text { Usulután } \\
\text { La Unión } \\
\text { San Francisco Gotera }\end{array}$ & $\begin{array}{c}\text { San Salvador } \\
\text { San Miguel } \\
\text { Nueva San Salvador } \\
\text { Usulután } \\
\text { La Unión } \\
\text { Cojutepeque }\end{array}$ \\
\hline
\end{tabular}

Fuente: elaboración propia. 
2. Participación electoral y ausentismo en las elecciones municipales de $\mathbf{2 0 0 0 .}$

Las elecciones municipales parecen tener menor importancia para los llamados analistas políticos. La mayor atención suele ser captada por las elecciones de ámbito nacional, ya sean éstas presidenciales, ya sean legislativas. No es extraño entonces que las cifras relativas a la participación y ausentismo se refieran al ámbito nacional. Contrario a este "imán de análisis" voy a realizar una primera aproximación a lo que ocurre en el ámbito municipal.

Como en el apartado anterior el análisis de la participación y la abstención lo limitaré al caso de las cabeceras departamentales. La información pertinente puede verse en el Cuadro № 6 . En éste, la participación absoluta viene dada por el total de votos (válidos, impugnados y nulos) ${ }^{17}$. Según este cuadro, la mayoría de cabeceras departamentales registraron una participación entre 30 y $40 \%$, aunque estuvieron más cercanas al límite inferior. La Unión es la cabecera con menor participación (29\%) mientras que Nueva San Salvador y Chalatenango están arriban del $42 \%$ siendo las más participativas.

\section{Cuadro N" 6 \\ Participación y Abstención en elecciones municipales -2000-}

\begin{tabular}{l|c|c|c|c|c}
\hline & & & \multicolumn{2}{|c|}{ Participación } & Abstención \\
\cline { 4 - 6 } Municipio & Población & Electorado & Abs. & \% & \% \\
\hline San Salvador & 479,605 & n.d. & 123,111 & n.d. & n.d. \\
Santa Ana & 248,963 & 149,412 & 46,462 & 31.1 & 68.9 \\
San Miguel & 239,038 & 122,930 & 37,939 & 30.9 & 69.1 \\
Nueva San Salvador & 158,207 & 84,589 & 35,599 & 42.1 & 57.9 \\
Usulután & 69,099 & 49,139 & 15,100 & 30.7 & 69.3 \\
Sonsonate & 96,772 & 62,440 & 20,877 & 33.4 & 66.6 \\
La Unión & 40,371 & 31,565 & 9,156 & 29.0 & 71.0 \\
Zacatecoluca & 62,352 & 44,986 & 16,147 & 35.9 & 64.1 \\
Chalatenango & 30,096 & 18,673 & 8,293 & 44.4 & 55.6 \\
Cojutepeque & 53,122 & 37,159 & 12,039 & 32.4 & 67.6 \\
Ahuachapán & 107,534 & 55,823 & 18,774 & 33.6 & 66.4 \\
San Francisco Gotẹra & 21,181 & 13,428 & 5,124 & 38.2 & 61.8 \\
San Vicente & 50,751 & 33,006 & 10,756 & 32.5 & 67.5 \\
Sensuntepeque & 41,068 & 24,157 & 7,392 & 30.6 & 69.4 \\
\hline
\end{tabular}

Fuentes: Población, en DIGESTYC (1996)

Electorado, en La Prensa Gráfica, 10 de marzo de 2000

Participación absoluta, en ECA № 617 
Para obtener una mejor imagen de la participación en elecciones municipales he elaborado el Cuadro $\mathrm{N}^{\mathrm{Q}} 7$ que recoge la participación efectiva ${ }^{18}$ para los años 1994, 1997 y 2000. Este cuadro muestra también las tasas de incremento o descenso de la participación efectiva para cada par de elecciones municipales. Como puede observarse, en general hubo una leve recuperación en la participación entre 1997 y 2000 . Salvo los municipios de Santa Ana, La Unión, Cojutepeque y San Vicente, en el resto se registró un incremento de votos. Sin embargo, dicho incremento no fue suficiente como para recuperar los niveles de votación alcanzados en 1994 (excepción hecha de Chalatenango y San Francisco Gotera). Por otro lado, las tasas de variación en la votación efectiva, en dirección del descenso, fueron bastante elevadas entre 1994 y 1997. Adicionalmente, llama la atención el caso de Chalatenango cuya tasa de descenso fue la menor para todos los municipios entre 1994 y 1997 y cuya tasa de incremento es la más alta entre 1997 y 2000. Habría que ver qué características tiene este municipio para explicar este comportamiento.

\section{Cuadro № 7}

\section{Participación efectiva local}

\begin{tabular}{l|r|r|r|r|r}
\hline \multirow{2}{*}{\multicolumn{1}{c|}{ Municipios }} & \multirow{2}{*}{1994} & \multicolumn{2}{c|}{1997} & \multicolumn{2}{c}{2000} \\
\cline { 3 - 6 } & Votos & Votos & T.V. & Votos & \multicolumn{1}{c}{ T.V. } \\
\hline San Salvador & 160,793 & 120,839 & -24.8 & 121,923 & +0.9 \\
Santa Ana & 67,085 & 51,668 & -23.0 & 45,495 & -11.9 \\
San Miguel & 40,989 & 35,597 & -13.2 & 37,230 & +4.6 \\
Nueva San Salvador & 40,262 & 31,306 & -22.2 & 35,059 & +12.0 \\
Usulután & 18,374 & 13,986 & -23.9 & 14,595 & +4.4 \\
Sonsonate & 27,702 & 19,676 & -29.0 & 20,413 & +3.7 \\
La Unión & 11,402 & 9,253 & -18.8 & 8,648 & -6.5 \\
Zacatecoluca & 18,350 & 15,197 & -17.2 & 15,756 & +3.6 \\
Chalatenango & 7,691 & 7,074 & -8.0 & 8,128 & +14.9 \\
Cojutepeque & 16,530 & 13,582 & -17.8 & 11,794 & -13.2 \\
Ahuachapán & 21,019 & 18,164 & -13.6 & 18,286 & +0.7 \\
San Francisco Gotera & 4,951 & 4,411 & -10.9 & 4,985 & +13.0 \\
San Vicente & 14,976 & 12,583 & -16.0 & 10,527 & -16.3 \\
Sensuntepeque & 9,174 & 6,801 & -25.9 & 7,115 & +4.6 \\
\hline
\end{tabular}

T.V. Tasa de Variación $=100 \times(\mathrm{V}-\mathrm{V}) / \mathrm{V}$.

Fuentes: elaboración propia sobre result'ados oficialcs

Votos de 1994 y 1997 en TSE (2000)

Votos de 2000 en ECA \#617. 


\section{Cuadro № 8}

Concentración municipal del voto legislativo departamental

\begin{tabular}{|c|c|c|c|c|c|c|c|c|c|}
\hline \multirow[b]{2}{*}{ Municipios } & \multicolumn{3}{|c|}{1994} & \multicolumn{3}{|c|}{1997} & \multicolumn{3}{|c|}{2000} \\
\hline & $\mathbf{A}$ & B & $\mathrm{C}$ & A & B & C & $\mathbf{A}$ & B & $\mathrm{C}$ \\
\hline San Salvador & 161,381 & 421,206 & 38.3 & 122,002 & 341,286 & 35.7 & 122,738 & 363,846 & 33.7 \\
\hline Santa Ana & 67,202 & 133,667 & 50.3 & 51,703 & 106,941 & 48.3 & 45,512 & 101.198 & 45.0 \\
\hline San Miguel & 40,950 & 83,605 & 49.0 & 35,515 & 76,741 & 46.3 & 37.182 & 84,830 & 43.8 \\
\hline Nueva San Salvador & 39,935 & 151,816 & 26.3 & 31,356 & 120,771 & 26.0 & 35,034 & 132,191 & 26.5 \\
\hline Usulután & 18,236 & 71,787 & 25.4 & 13,933 & 60,874 & 22.9 & 14,543 & 71,297 & 20.4 \\
\hline Sonsonate & 27,643 & 106,043 & 26.1 & 19,702 & 82,927 & 23.8 & 20,452 & 91.377 & 22.4 \\
\hline La Unión & 11,349 & 45,969 & 24.7 & 9,269 & 39,753 & 23.3 & 8,571 & $43,4 n 2$ & 19.7 \\
\hline Zacalecoluca & 18,221 & 68,329 & 26.7 & 15,323 & 59,341 & 25.8 & 15,806 & 66,196 & 23.9 \\
\hline Chalatenango & 7,686 & 50,252 & 15.3 & 7,040 & 44,879 & 15.7 & 8,161 & 50,882 & 16. \\
\hline Cojulepcque & 16,534 & 48,602 & 34.0 & 13,542 & 41,731 & 32.5 & 11,802 & 44,960 & 26 \\
\hline Ahuachapán & 20,980 & 60,388 & 34.7 & 18,1159 & 53,087 & 34.0 & 18,406 & 58.223 & 31. \\
\hline San Francisco Golera & 4,921 & 35,812 & 13.7 & 4,409 & 33,410 & 13.2 & 4,927 & 39,817 & 12 \\
\hline San Vicenle & 14,971 & 37,426 & 40.0 & 12,531 & 32,805 & 38.2 & 10,487 & 32,804 & 32.0 \\
\hline Sensuntepeque & 9,085 & 30,375 & 29.9 & 6,801 & 25,057 & 27.1 & 7,107 & 29.246 & 24.3 \\
\hline
\end{tabular}

A = Votos legislativos del municipio.

B = Votos legislativos del departamento.

$\mathrm{C}=$ Concentración municipal del voto legislativo, $100 \times \mathrm{A} / \mathrm{B}$.

Fuentes: elaboración propia sobre resultados oficiales

Votos de 1994 y 1997, en TSE (2000)

Votos de 2000, en ECA \# 617.

Finalmente el Cuadro № 8 registra la concentración del voto legislativo en cada una de las cabeceras departamentales. El objetivo de presentar este cuadro consiste en determinar la importancia que estos municipios tienen para los resultados en las elecciones legislativas. Se supone que cuanto mayor sea la concentración del voto legislativo en el municipio en cuestión, mayor importancia tendrá éste para los partidos. Municipios como Santa Ana y San Miguel, por ejemplo, han concentrado a lo largo del período más del $40 \%$ de los votos necesarios para obtener un escaño legislativo en sus respectivos departamentos. En el otro extremo, Chalatenango y San Francisco Gotera han registrado unos niveles de concentración del voto legislativo por debajo del $15 \%$. Esto no quiere decir que sean los municipios de menor importancia dentro de sus respectivos departamentos. La menor concentración puede deberse, especialmente en el caso de Chalatenango, a la cantidad de municipios (33) que hay en el departamento, lo que favorece la dispersión del voto legislativo entre todos estos municipios. Lo mismo vale para Gotera pues en Morazán el voto legislativo se reparte entre 26 municipios. 


\section{Evolución del control partidista sobre los gobiernos municipales.}

Otra dimensión que puede resultar interesante para el análisis del comportamiento electoral municipal viene dada por el control de los gobiernos municipales por partidos, en la medida en que dicho control expresa las preferencias mayoritarias de los electores en los municipios respectivos. Al hacer este análisis a lo largo de varios periodos electorales puede obtenerse una imagen sobre la estabilidad de estas preferencias mayoritarias. Por ejemplo, el control del gobierno de un municipio dado por determinado partido durante varios periodos puede interpretarse como la satisfacción del electorado con la gestión del partido en dicho gobierno. Según el Cuadro №9, esta situación estaría ocurriendo en San Vicente y Sensuntepeque en tanto que, para el periodo democrático iniciado en 1994, ARENA ha ganado consecutivamente.

El Cuadro № 9

Control de Concejos Municipales, según partido, 1994-2000-07-03

\begin{tabular}{l|ccc}
\hline \multirow{2}{*}{ Municipio } & \multicolumn{3}{|c}{ Partido o Coalición electoral } \\
\cline { 2 - 4 } & 1994 & 1997 & 2000 \\
\hline \multirow{2}{*}{ San Salvador } & ARENA & FMLN-CD-MU & FMLN-USC \\
Santa Ana & ARENA & FMLN-CD-MU & FMLN-USC \\
San Miguel & ARENA & ARENA & PDC \\
Nueva San Salvador & ARENA & FMLN & FMLN \\
Usulután & ARENA & ARENA & PCN \\
Sonsonate & ARENA & FMLN-CD & FMLN \\
La Unión & PDC & PDC & ARENA \\
Zacatecoluca & ARENA & FMLN & FMLN \\
Chalatenango & ARENA & FMLN & FMLN \\
Cojutepeque & ARENA & PRSC & ARENA \\
Ahuachapán & ARENA & ARENA & FMLN PD- \\
San Francisco Gotera & ARENA & ARENA & FMLN-CD \\
San Vicente & ARENA & ARENA & ARENA \\
Sensuntepeque & ARENA & ARENA & ARENA \\
\hline
\end{tabular}

Fuente: elaboración propia sobre resultados oficiales.

Por otro lado, considerando las catorce cabeceras en conjunto, ARENA ha venido perdiendo el control de municipios entre 1994 y 2000. De gobernar 13 cabeceras ha pasado a gobernar 4 , en 2000 . Un movimiento inverso se registra con relación a los municipios donde el FMLN goza de preferencias mayoritarias. De no gobemar cabecera alguna en 1994 ha pasado a ejercer el gobierno en 8 cabeceras. Aunque hay que hacer constar que en tres de estas ocho cabeceras, el 
FMLN comparte el gobierno con otros partidos. Finalmente, el PDC ha logrado controlar una alcaldía en cada proceso electoral y el $\mathrm{PCN}$ le arrebató la alcaldía de Usulután al partido ARENA en $2000^{19}$.

\section{Expectativas y ofertas electorales.}

Uno de los rotativos nacionales de mayor circulación publicó en un suplemento dedicado al proceso electoral 2000 una serie de problemas que aquejaban a la población de cada uno de los departamentos del país. Entre estos problemas se puede mencionar: contaminación ambiental, desorden comercial, deforestación, basura, delincuencia, congestionamiento vehicular, falta de lugares recreativos, mala red de caminos vecinales y necesidad de mercados municipales (La Prensa Gráfica, 11 de marzo de 2000). Algunos de estos problemas, si no todos, aparecen a los ojos de los electores como sin solución. Sin embargo, el que estos problemas aparezcan de este modo puede tener diversas explicaciones. Para algunos electores se trata de problemas que exigen recursos de cuyo monto no disponen los gobiernos municipales. Para otros, se trata de problemas en los que es necesaria una acción concertada de varios concejos municipales que comparten tales problemas. Finalmente, para otros el "problema" no está en los "problemas" sino en quienes dicen que van a resolverlos (los candidatos o candidatas, y los partidos) siendo que una vez "en el poder" se olvidan de las promesas hechas" En la medida en que finalmente no son resueltos los problemas que aquejan a la ciudadanía, estos últimos electores reafirman su convicción de que el problema está en los políticos ${ }^{21}$. Es probable que este grupo de electores vaya creciendo y esté en la base de quienes, de elección a elección, se van ausentando de las urnas o centros de votación ${ }^{22}$.

Este tipo de problemáticas han sido abordadas por diversos analistas y medios. No voy a insistir aquí en este asunto. Quisiera más bien llamar la atención sobre un punto que me parece importante tratar. En el ámbito de las elecciones legislativas ha salido a luz pública bajo la denominación de candidaturas independientes. Varios ciudadanos intentaron ser inscritos como candidatos a diputados sin respaldo de partido político. Esto, según lo señala el Código Electoral no es posible. El Código establece el monopolio de la representación política para los partidos políticos. Sin embargo, la iniciativa de estos ciudadanos no debería ser desechada "hasta nuevo aviso". Valdría la pena discutir la posibilidad de las candidaturas independientes en los niveles locales. Al final de cuentas, en estos niveles es más probable que se de un contacto directo entre candidatos y ciudadanos. Dicho de otra forma, el control de la ciudadanía sobre la gestión gubernamental de sus representantes puede resultar más viable en los municipios, especialmente si éstos son pequeños, si se hace depender la reelección de las autoridades municipales directamente del juicio de los electores. 
Por otra parte, no estoy planteando la sustitución de las candidaturas partidistas por candidaturas independientes. Más bien se trata de ofrecer una mayor opción (y tal vez en mejores condiciones de selección) a los electores. Esta medida no es nada nueva en la experiencia electoral centroamericana. En Guatemala y Nicaragua ya hace varios años se permitió siempre que la candidatura estuviese apoyada por una Asociación o Comité Cívico. La experiencia en este nivel podría convertirse posteriormente en criterio de decisión para permitirla o no en el nivel de las candidaturas legislativas.

En el fondo aquí hay un problema que tiene que ver, por un lado, con el acercamiento o distanciamiento de los representantes y la ciudadanía, y por otro lado, con la mediación de los partidos entre representantes y ciudadanos. Se supone que cuanto mayor es el tamaño del municipio, la relación representanteciudadanía tiende a ser menor. El partido media esta relación y sirve de criterio a la segunda para decidir sobre el primero. La bandera del partido tiende a pesar más que la biografía del que se postula a representante. El partido, que se supone se convierte en elemento racional de decisión a través de su programa, orienta la decisión del elector. Los partidos políticos están llamados a resumir en sus plataformas electorales las soluciones técnicas que ofrecen a los problemas que aquejan a la ciudadanía. Los partidos gozan de más recursos para preparar las propuestas de solución que ofrecen a los electores en el intercambio político. Sin embargo, habría que examinar si así funciona realmente la política partidista y el comportamiento de los electores. Si las cosas no siguen la lógica planteada ¿cuáles son los argumentos a favor del monopolio de la representación política por parte de los partidos al nivel municipal?

Finalmente, si los partidos que compiten en el ámbito municipal son los mismos que compiten en el ámbito nacional se corre el riesgo de que sea la política nacional la que predomine sobre la política municipal en la orientación de los partidos. Éstos pueden buscar votos legislativos a través de los votos municipales, especialmente en el caso de los votantes que no siguen la lógica del voto cruzado. Ofreciendo un "buen concejo municipal" se puede estar intentando arrastrar votos legislativos hacia el propio partido. Al final de cuentas, uno de los objetivos fundamentales de los partidos es obtener el número de votos que le asegure el máximo poder. $Y$ el máximo poder en un sistema presidencialista como el nuestro va de lo nacional a lo municipal.

\section{Referencias Bibliográficas}

Artiga-González, Álvaro (2000). "Electores, partidos y la caja de Pandora", en ECA \# 617, Pp.267-287.

DIGESTYC (1996). Proyección de la población de El Salvador. San Salvador: Dirección General de Estadística y Censos, Ministerio de Economía.

ECA \# 617. Documentación, Pp.329-360).

La Prensa Gráfica, 10 de marzo de 2000. 
La Prensa Gráfica, 11 de marzo de 2000, Pp.43-54.

TSE (2000). Guía elección 2000. San Salvador, Edit-TSE.

Zavala, Claudia (2000). “¿Votaciones? ¡No, gracias!”, en La Prensa Gráfica, 13 de marzo de 2000, P.42.

\section{Notas}

1. El análisis electoral que aquí realizo para el nivel municipal se refiere únicamente a las cabeceras de los 14 departamentos. Esta es otra de las razones por las cuales considero que los hallazgos deben considerarse como nuevas hipótesis sobre las que hay que profundizar.

2. Es decir, cualquier ciudadano podría votar por dos partidos o coaliciones diferentes según sus preferencias partidistas para la elección municipal y nacional.

3. De aquí en adelante utilizaré los términos "municipal" y "local" de manera indistinta. Ambos hacen referencia a las elecciones de concejos municipales. El término "nacional" lo empleo para referirme a la elección de autoridades nacionales. Aunque los diputados se eligen en circunscripciones departamentales forman parte de un "poder nacional".

4. La discusión sobre si el electorado vota por el partido o por la candidatura es otra manera de aproximarse el fenómeno de una o dos lógicas de votación. Este fenómeno se da no sólo cuando hay simultaneidad electoral sino también cuando no la hay.

5. Por supuesto que esto llevaría a una discusión más sustantiva respecto de sí una acción de este tipo representaría una limitación o restricción más para la decisión de los electores.

6. El Código electoral vigente no contempla la existencia de este tipo de organizaciones partidarias. Esto supone un monopolio de la representación política a favor de los partidos de ámbito nacional. Uno puede preguntarse por la autonomía de las candidaturas y partidos locales respecto de los intereses nacionales de los partidos de ámbito nacional.

7. La selección de los años 1994,1997 y 2000 obedece a que sólo para estos años se puede hablar de elecciones libres y competitivas. Aunque la serie de elecciones más o menos libres y limpias, con respeto de los resultados, puede fecharse desde 1982 con la elección de Asamblea Constituyente, elecciones competitivas (sin restricción de partido político alguno) sólo han sido estas tres.

8. No resulta extraño el comportamiento observado en este año si tomamos en cuenta el efecto "Silva" sobre la decisión de los electores. Aquí estariamos muy probablemente ante un voto cruzado. Simpatizantes de otros partidos, en elecciones nacionales, dieron su voto al FMLN en elecciones locales.

9. Medida a partir de los votos válidos.

10. Hay quienes sostienen que el nuevo régimen se habria instaurado con la Constitución Política de 1983. Considero que lo que se observa desde el golpe de estado de 1979 es más bien una transición política prolongada que combina elecciones y conflicto bélico interno y que termina justamente con las reformas a la Constitución de 1983, que permiten la participación política legal de la oposición armada aglutinada en el FMLN. El contexto de diảlogo y negociación en el que se pactan dichas reformas desemboca en la firma de los Acuerdos de Paz, cuyo cumplimiento 
es precisamente la instauración del régimen diseñado en tales Acuerdos. Todos los procesos electorales desde 1982 hasta 1991 se llevan a cabo en contexto de guerra. Formaron parte de la estrategia contrainsurgente de los Estados Unidos. Lo cual no debería llevar a la negación de algún aporte a la posterior pacificación y transición política.

11. Mientras los votos locales deciden la elección de las autoridades municipales, los votos nacionales registrados en las cabeceras deben ser agregados a los votos nacionales del resto de municipios del departamento en cuestión.

12. Este análisis podría completarse con una perspectiva temporal al estudiar lo que ocurre en cada municipio en diferentes años electorales. Al proceder asi podría establecerse el predominio o no de determinada lógica de votación por municipio. Razones de espacio me impiden realizar dicho análisis aquí.

13. Esta situación podría sugerir que los caudales electorales de los partidos en el nivel municipal se nutren de votos de electores que no necesariamente se identifican con tales partidos. Podríamos hablar en este caso de un arrastre municipal del voto.

14. Esta situación podría sugerir que los caudalcs clectorales de los partidos en el nivel nacional se alimentan de votos provenientes de electores que no neccsariamente se identifican con tales partidos. Podriamos, entonces, hablar de un arrastre nacional del voto.

15. El indice de heterogeneidad del voto pucde calcularse mediante la expresión: HcV $=\Omega$ Vloc - Vnac $\Omega \times 100 /$ Vmedio, donde Vloc es el total de votos locales; Vnac, cl total de votos nacionales y Vmedio es el promedio de los dos anteriores.

16. Recomiendo al lector que sc auxilic del Cuadro $N^{2} 4$ para lecr correctamente cl Cuadro N 55 .

17. Debe diferenciarse el ausentismo y el abstencionismo. El primero se refierc a la no concurrencia de los electores a los centros de votación. El segundo incluye a quicnes no van a votar (quicnes se ausentan) y quienes una vez en los centros de votación emiten un voto en blanco. Esto quicre decir que el abstencionismo es siempre mayor que el ausentismo.

18. Medida, en términos absolutos, por los votos válidos en cada municipio.

19. Un análisis de los apoyos territoriales de los partidos al nivel municipal pucde versc en Artiga-González (2000).

20. Interrogado por Claudia Zavala (2000), un cmplcado de 31 años que no acude a votar responde: "Cuando estuve metido en cso, yo creía que de verdad sc podían cambiar las cosas. Ahora sć que lo único que esa gente busca cs poder...cso no cs democracia. Y yo ya estoy harto de lo mismo".

21. En este sentido resulta muy atinada la afirmación de Claudia Zavala (2000) cuando dice: "La indiferencia, la deccpción y la incredulidad pudicron más que la rcsponsabilidad ciudadana. El radiante sol y cl olor a pescado frito fue tentador para muchos". Recordar que las clecciones sc celcbran en cl país en los mescs más calurosos del año,'meses en los que cualquicr "vacación" pucde scr aprovechada para darse una cscapada al mar.

22. Otro grupo de electores se ausenta de los centros de volación por los obstáculos que tiene que superar, como por cjemplo, la búsqueda del lugar donde les toca votar, cl transportc hacia los centros de votación, cl tener que contar con un carnć para poder volar, ctc. 